\title{
Surgical management of macular holes: results using gas tamponade alone, or in combination with autologous platelet concentrate, or transforming growth factor $\beta 2$
}

\author{
M Minihan, M Goggin, P E Cleary
}

\begin{abstract}
Background-Vitrectomy and gas tamponade has become a recognised technique for the treatment of macular holes. In an attempt to improve the anatomic and visual success of the procedure, various adjunctive therapies-cytokines, serum, and platelets-have been employed. A consecutive series of 85 eyes which underwent macular hole surgery using gas tamponade alone, or gas tamponade with either the cytokine transforming growth factor $\beta 2$ (TGF- $\beta 2$ ) or autologous platelet concentrate is reported.
\end{abstract}

Methods-Twenty eyes had vitrectomy and $20 \% \mathrm{SF}_{6}$ gas tamponade; 15 had vitrectomy, $20 \% \mathbf{S F}_{6}$ gas, and TGF- $\beta 2 ; 50$ had vitrectomy, $16 \% \mathrm{C}_{3} \mathrm{~F}_{8}$ gas tamponade, and $0.1 \mathrm{ml}$ of autologous platelet concentrate prepared during the procedure.

Results-Anatomic success occurred in $86 \%$ of eyes, with $96 \%$ of the platelet treated group achieving closure of the macular hole. Visual acuity improved by two lines or more in $65 \%$ of the $\mathrm{SF}_{6}$ only group, $33 \%$ of those treated with TGF- $\beta 2$, and in $74 \%$ of the platelet treated group. In the platelet treated group $40 \%$ achieved $6 / 12$ or better and $62 \%$ achieved $6 / 18$ or better. The best visual results were obtained in stage 2 holes.

Conclusion-Vitrectomy for macular holes is often of benefit and patients may recover good visual acuity, especially early in the disease process. The procedure has a number of serious complications, and the postoperative posturing requirement is difficult. Patients need to be informed of such concerns before surgery.

(Br f Ophthalmol 1997;81:1073-1079)

Ophthalmology, Cork University Hospital and University College Cork, Wilton, Cork, Ireland

M Minihan

$M$ Goggin

P E Cleary

Correspondence to: Ms Miriam Minihan,

Department of

Ophthalmology, Cork

University Hospital, Wilton,

Cork, Ireland.

Accepted for publication 13 August 1997 macula, and that this dehiscence is due to tractional forces on the macula by the prefoveal vitreous cortex, and by epiretinal membranes, especially in a tangential manner.

Before the use of vitrectomy for the treatment of macular holes, it was believed that a technique which would promote flattening of the small rim of retinal detachment surrounding the macular hole, would inevitably lead to an improvement in visual acuity. Spontaneous flattening has been reported in rare circumstances. ${ }^{3}$ Laser photocoagulation was employed in an attempt to flatten this detached area, ${ }^{4}$ but its destructive properties have precluded its use in this vision sensitive area.

Macular hole surgery has become defined as an accepted technique since Kelly and Wendel ${ }^{5}$ first reported the benefit of pars plana vitrectomy and gas tamponade in inducing closure of macular holes, flattening of the neurosensory rim, and improvement in visual acuity. Since then, a variety of techniques have been reported in an attempt to improve the anatomic and visual results. These include the use of wound healing substances, cytokines, to promote closure of the macular hole. Glaser et $a l^{6}$ reported a $90-100 \%$ success rate in the closure of macular holes using the cytokine transforming growth factor $\beta 2$ (TGF- $\beta 2$ ).

Other growth promoting substances, such as serum $^{7}$ and platelets, ${ }^{8}$ have been used in an attempt to improve the anatomic and visual success of macular hole surgery. However, the clinical decision of when and if to operate is still not clearly defined. ${ }^{9}$ Some recommend surgery for uniocular cases only. ${ }^{10}$ Nevertheless, impressive visual results have been reported in some series, although it is not yet possible to identify preoperatively those patients capable of worthwhile visual improvement.

We report a consecutive series of 85 eyes, and document results and complications in an attempt to better define indications for surgery. We describe three groups - those treated with vitrectomy and gas tamponade only; those treated with vitrectomy, gas tamponade, and TGF- $\beta 2$; and a prospective trial of vitrectomy, gas tamponade, and autologous platelet concentrate.

Subjects and methods

We report on 85 procedures in 75 patients. Two patients had both eyes operated, and eight procedures were reoperations. Patients were included in the study if they had a visual acuity 
of $6 / 18$ or worse, no other significant ocular disease, and had symptoms for less than 1 year. Preoperatively, a complete ocular examination was performed, including best corrected Snellen visual acuity, intraocular pressure measurement, assessment of lens clarity, slit lamp biomicroscopy, contact lens examination of the fundus, and fundus fluorescein angiography. All patients had preoperative confrontational visual field examination and patients in the platelet treated group had suprathreshold Humphrey visual field testing.

Fifty nine $(79 \%)$ patients were female and $16(21 \%)$ male. They ranged in age from 23 to 85 , mean 65 years. The macular holes were staged according to the criteria outlined by Gass. ${ }^{211}$ There were $20(23.5 \%)$ stage 2, 45 $(53 \%)$ stage 3 , and $20(23.5 \%)$ stage 4 holes. This staging was confirmed at the time of surgery. There was no statistical difference in the proportions of different stages in the different treatment groups. One case was a traumatic macular hole, all others were idiopathic. Preoperative visual acuity ranged from $6 / 18$ to $1 / 60$. All patients received an information leaflet preoperatively, and the importance of postoperative posturing was reiterated during their inpatient stay.

Three port trans pars plana vitrectomy was performed in all eyes. In stage 2 and 3 holes, the posterior hyaloid was detached and vitreous removed as far out to the periphery as possible. In stage 4 holes, posterior vitreous detachment was confirmed using aspiration with a silicone tipped needle. Before air/fluid exchange, all patients underwent careful examination of the peripheral fundus to discover any induced retinal tears. Following air/fluid exchange, 10 minutes were allowed for peripheral fluid to drain posteriorly. This fluid was then aspirated and an air-gas exchange was performed. The intraocular gas used was either $20 \% \mathrm{SF}_{6}$ or $16 \% \mathrm{C}_{3} \mathrm{~F}_{8}$ depending on which group the patient fell into.

Twenty eyes were treated with $20 \% \mathrm{SF}_{6}$ gas tamponade alone. Patients then postured prone for 7-10 days. Fifteen eyes received $1000 \mathrm{ng}$ of porcine TGF- $\beta 2$ in $0.1 \mathrm{ml}$ of balanced salt solution under air at the macula, followed by air/gas exchange using $20 \% \mathrm{SF}_{6}$ gas. These patients postured supine for 6 hours then prone for at least 10 days. Fifty eyes had $0.1 \mathrm{ml}$ of autologous platelet concentrate placed on the macula followed by air $/ 16 \% \mathrm{C}_{3} \mathrm{~F}_{8}$ gas tamponade. These patients postured supine for 6 hours, then prone for 2-4 weeks.

In the $\mathrm{SF}_{6}$, and $\mathrm{SF}_{6}+\mathrm{TGF}-\beta$ groups, fluid was drained through the hole and a search was made for epiretinal membranes around the hole with a membrane pick, sometimes producing nerve fibre layer haemorrhages. In the $\mathrm{C}_{3} \mathrm{~F}_{8}+$ platelets group, which formed a prospective trial, we followed a standard procedure as proposed by Glaser in which fluid was not drained and no membranes were peeled.

Platelets were prepared in the manner described by Gaudric et $a l^{8}{ }^{\text {Immediately }}$ before the procedure, $40 \mathrm{ml}$ of venous blood was taken from the patient. This was mixed gently with $6 \mathrm{ml}$ of acid citrate dextrose
(ACD). The mixture was then centrifuged at $280 \mathrm{~g}$ for 15 minutes and the platelet rich plasma decanted. The plasma was mixed with $1 / 8$ volume ACD and further centrifuged at $1000 \mathrm{~g}$ for 10 minutes. The packed platelets were then mixed with $0.6 \mathrm{ml}$ of sodium chloride to achieve a platelet suspension. The preparation of platelets was performed entirely in the operating theatre.

Patients were advised to continue to posture at home for up to 4 weeks postoperatively, and were contacted by telephone to encourage posturing.

All patients have a minimum follow up of 6 months. Postoperative assessment was carried out at $1,3,6$, and 12 months postoperatively, and included best corrected Snellen visual acuity, intraocular pressure measurement, assessment of lens clarity, slit lamp biomicroscopy, contact lens examination of the fundus, and suprathreshold Humphrey visual field examination.

Results were analysed statistically using Student's $t$ testing and $\chi^{2}$ analysis. Visual acuity measurements were converted to $\log$ MAR to compare means.

\section{Results}

Postoperatively, there was zero to mild anterior chamber activity. In the platelet treated group, in the immediate postoperative period, a white 'coagulum' was visible over the posterior pole and covering the macular hole, and this material slowly disappeared over the subsequent 1-2 weeks.

Cases were deemed an anatomic success if the subretinal fluid surrounding the hole resolved and the retina flattened. The macular hole was closed in $15(75 \%)$ eyes treated with $\mathrm{SF}_{6}$ gas alone; in $10(66 \%)$ eyes treated with $\mathrm{SF}_{6}$ gas and TGF- $\beta 2$; and in $48(96 \%)$ eyes treated with $\mathrm{C}_{3} \mathrm{~F}_{8}$ gas and platelet concentrate (Table 1). The rate of anatomic success in the $\mathrm{C}_{3} \mathrm{~F}_{8}+$ platelet concentrate group was significantly better than either the $\mathrm{SF}_{6}+\mathrm{TGF}-\beta$ group (Fisher's exact: $\mathrm{p}=0.017$, confidence interval (CI) $0.01-0.89$ ), or the $\mathrm{SF}_{6}$ only group (Fisher's exact: $p=0.005, \quad \mathrm{CI}: 0.01-0.63$ ). Eighteen (90\%) stage 2, $39(86 \%)$ stage 3, and $16(80 \%)$ stage 4 holes were an anatomic success. There was no statistical difference in the anatomic success rates of the different stages.

Table 1 Anatomic results

\begin{tabular}{lcccl}
\hline & $\begin{array}{l}\text { Closed, no } \\
\text { edge }\end{array}$ & $\begin{array}{l}\text { Flat, with } \\
\text { edge }\end{array}$ & Open & Total \\
\hline $\mathrm{SF}_{6}$ & 10 & 5 & 5 & 20 \\
$\mathrm{SF}_{6}+\mathrm{TGF}-\beta$ & 6 & 4 & 5 & 15 \\
$\mathrm{C}_{3} \mathrm{~F}_{8}+$ platelets & 46 & 2 & 2 & 50 \\
Total & 62 & 11 & 12 & 85
\end{tabular}

$\mathrm{C}_{3} \mathrm{~F}_{8}+$ platelets $v \mathrm{SF}_{6}+/-\mathrm{TGF}-\beta 2$ : better surgical results $\mathrm{p}=0.01$.

Table 2 Visual outcome of different staged holes

\begin{tabular}{llllr}
\hline & $\begin{array}{l}\text { 6/12 or } \\
\text { better }\end{array}$ & $\begin{array}{l}\text { 6/18 or } \\
\text { better }\end{array}$ & $\begin{array}{l}\text { VA increase, } \\
\text { 2 lines or } \\
\text { more }\end{array}$ & $\begin{array}{l}\text { VA increase, 4 } \\
\text { lines or more }\end{array}$ \\
\hline Stage 2 & 12 & 11 & 14 & 4 \\
Stage 3 & 14 & 24 & 29 & 12 \\
Stage 4 & 3 & 5 & 10 & 5 \\
\hline
\end{tabular}




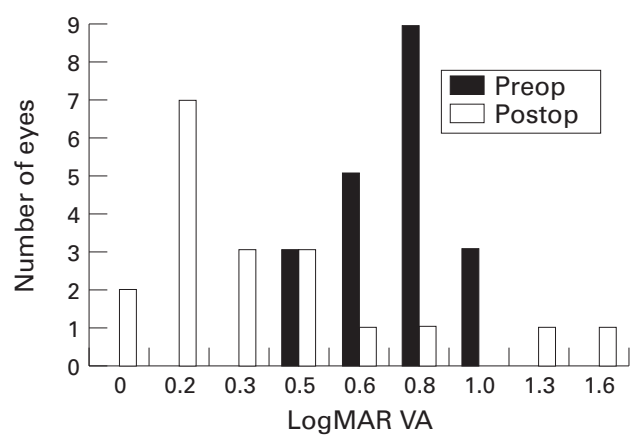

Figure 1 Pre and postoperative $\log M A R$ visual acuity of stage 2 holes.

Postoperative visual acuity ranged from $6 / 6$ to counting fingers. Best visual results were in those eyes with stage 2 holes, $60 \%$ of these eyes achieved final visual acuity of $6 / 12$ or better. Visual acuity improved by two lines or more in $14(70 \%)$ stage $2,29(64 \%)$ stage 3 , and 10 $(55 \%)$ stage 4 holes (Table 2). Mean logMAR visual acuity postoperatively was better (Student's $t$ test) in stage 2 holes than either stage 3 $(p=0.02)$ or stage $4 \quad(p=0.004)$ holes. Of successful cases, stage 2 holes are more likely than either stage 3 (Fisher's exact: $p=0.012$ ) or stage 4 (Fisher's exact: $p=0.004$ ) to achieve a final visual acuity of $6 / 9$ or better (Fig 1). Overall, $36 \%$ of eyes had visual acuity of $6 / 12$ or better postoperatively, and $54 \%$ had $6 / 18$ or better. Visual acuity improved by two lines or more in $13(65 \%)$ of eyes which had $\mathrm{SF}_{6}$ gas tamponade alone, in five $(33 \%)$ eyes with $\mathrm{SF}_{6}$ gas and TGF- $\beta 2$; and in $37(74 \%)$ of the platelet treated group (Table 3). Rate of two line improvement in the platelet treated group was significantly better than in the $\mathrm{SF}_{6}+\mathrm{TGF}-\beta 2$ group $\left(\chi^{2}: p=0.0004, C I: 0.03-0.44\right)$, but no significant difference existed between the $\mathrm{C}_{3} \mathrm{~F}_{8}$ + platelet group and the $\mathrm{SF}_{6}$ only group $\left(\chi^{2}\right.$ : $\mathrm{p}=0.6$, CI: 0.19-2.39). At all levels visual results were worse in the TGF- $\beta 2$ treated group.

Eight procedures were reoperations for previously failed surgery. Although the anatomic success rate of these procedures was $75 \%$ (six of eight), only two (25\%) improved by two lines or more in visual acuity. Best visual acuity after a second procedure was $6 / 24$, and this group of eyes had significantly worse visual results than those with one operation only $(\mathrm{p}<0.001)$. Five eyes $(6 \%)$ developed retinal tears intraoperatively, three $(3 \%)$ developed rhegmatogenous retinal detachments postoperatively. Three eyes had splinter haemorrhages in the nerve fibre layer at the optic disc intraoperatively. In two eyes (2\%), a previously closed macular hole reopened. One eye had optic atrophy postoperatively.

Twenty two patients have been found to have temporal or inferotemporal visual field loss

Table 3 Visual results

\begin{tabular}{lcccc}
\hline & $6 / 12$ or better & $6 / 18$ or better & $\begin{array}{l}\text { VA increase, 2 lines } \\
\text { or more }\end{array}$ & $\begin{array}{c}\text { VA increase, } 4 \\
\text { lines or more }\end{array}$ \\
\hline $\mathrm{SF}_{6}$ & $9(45 \%)$ & $12(60 \%)$ & $13(55 \%)$ & $4(20 \%)$ \\
$\mathrm{SF}_{6}+\mathrm{TGF}-\beta$ & $2(13 \%)$ & $3(60 \%)$ & $5(33 \%)$ & $2(13 \%)$ \\
$\mathrm{C}_{3} \mathrm{~F}_{8}+$ platelets & $21(43 \%)$ & $31(63 \%)$ & $36(73 \%)$ & $20(42 \%)$ \\
\hline
\end{tabular}

postoperatively. All three eyes with optic disc haemorrhage during surgery had postoperative visual field loss. Of those with visual field loss, six had $\mathrm{SF}_{6}$ gas tamponade and 16 had $\mathrm{C}_{3} \mathrm{~F}_{8}$ gas tamponade, 17 required posterior hyaloid stripping (stage 2 or stage 3 ), and five already had posterior vitreous detachment (stage 4). Lens opacities have progressed to cause significant cataracts in 33 patients to date. Abnormalities were present at the macula in a number of eyes with an anatomically successful outcome. Twelve (14\%) had radial striae emanating from the closed hole, which tended to disappear after about 6 weeks. There was no significant difference in visual outcome in eyes with or without radial striae. Focal retinal pigmentary changes, most commonly a pigment spot was present in $20(24 \%)$ eyes. This pigmentary change was more likely to occur in the platelet treated group than in either of the other groups $(p=0.048$ and $p=0.025)$. Mean visual outcome was not significantly different between eyes with or without this pigment hypertrophy. Ten $(12 \%)$ eyes had fine epiretinal membranes, not causing distortion or affecting vision, postoperatively.

In 11 eyes the edge of the macular hole was still visible postoperatively, despite closure of the hole. This edge was less likely to be present in the platelet treated group than in either of the other two groups (Fisher's exact: $p=0.01$, CI: $0.001-0.62$ ). Eyes without an edge were more likely to achieve at least four lines of improvement in visual acuity than eyes with an edge (Fisher's exact: $\mathrm{p}=0.01, \mathrm{CI}: 0.005-1.64$ ).

\section{Discussion}

The goal of surgical therapy for macular hole is closure of the hole, and flattening of the cuff of subretinal fluid surrounding the hole with accompanying visual improvement. Recent years have seen the search for techniques to improve hole closure, without damage to surrounding retinal tissue, thus increasing the chance of substantial visual recovery.

Kelly and Wendel $^{5}$ first described vitreous surgery for macular holes. Our initial technique was similar to theirs in that we performed vitrectomy, $\mathrm{SF}_{6}$ gas tamponade, and postoperative posturing. Others have proposed techniques such as aggressive peeling of very fine epiretinal membranes, and even peeling of the internal limiting membrane (ILM) and drainage of subretinal fluid through the macular hole. As our experience with the procedure increased, we undertook a prospective study using a standard technique in which we avoided any contact with the macular hole or the retina surrounding the hole. Epiretinal membranes were peeled only if easily visible or causing retinal traction. We have noted fine epiretinal membranes at the macula, usually contracted postoperatively, but they have not interfered with vision or caused traction. It is, perhaps, these membranes that others are peeling during surgery. It seems possible therefore to achieve a high anatomic success rate despite avoiding aggressive peeling of fine ERMs or of the ILM which may cause nerve fibre layer haemorrhages. Glaser's patients also achieved a 
high anatomic success rate without foveal manipulation. ${ }^{12}$ In our study, the three different treatment groups were treated consecutively, which may introduce a learning curve bias, although we believe that this was not significant as the surgical procedure is relatively straight forward.

There is evidence that the duration of the intraocular gas bubble tamponade may have an effect on the outcome of macular hole surgery. ${ }^{13}$ We initially used $\mathrm{SF}_{6}$ gas but, for our prospective trial, changed to the longer acting $\mathrm{C}_{3} \mathrm{~F}_{8}$ gas. We also encouraged our patients to posture by informing them of the requirement in a patient information leaflet, by reiterating this information verbally postoperatively, and telephoning them at home. Posturing remains a troublesome and unpleasant requirement for patients.

Adjunctive therapy was introduced to the surgical management of macular holes by Glaser, in an attempt to improve the anatomic and visual outcomes. The rationale for the use of cytokines is to promote chorioretinal adhesion at the margins of the hole, and it has been suggested $^{6}$ that cytokines may also have a growth promoting effect, stimulating photoreceptor regeneration. Other sources known to be rich in cytokines including serum and platelets, and thrombin have now also been used as adjuncts. Platelets are a rich source of cytokines, and this resource has been exploited to promote wound healing. ${ }^{14}$ Gaudric and colleagues $^{8}$ suggested in a preliminary report that an autologous platelet preparation is beneficial in treating macular holes and others have reported the successful use of platelets in a small number of cases. ${ }^{15}$ Smiddy et al have shown closure of induced retinal tears, in rabbit eyes, using the cytokine TGF- $\beta 2,{ }^{16}$ and autologous serum has been shown to promote closure of experimental retinal tears in the rabbit retina. ${ }^{17}$

Several studies in which a variety of techniques were used have now reported an anatomic success rate of $80-90 \%$ in macular hole surgery. Kelly and Wendel used vitrectomy, and $\mathrm{SF}_{6}$ gas tamponade alone to achieve $58 \%$ anatomic success. ${ }^{5}$ This had improved to $90 \%$ anatomic success in a larger series of patients. ${ }^{18}$ Glaser and colleagues achieved $100 \%$ macular hole closure in patients treated with $1330 \mathrm{ng}$ dose of TGF- $\beta 2 .{ }^{6}{ }^{19}$ Liggett and coworkers $^{7}$ found $100 \%$ anatomic success in 11 eyes treated with human autologous serum adjunct to vitrectomy and gas tamponade. In 1995 Gaudric et $a l^{8}$ reported $95 \%$ anatomic success in 20 eyes, and in 1996 Korobelnik et $a l^{15}$ reported $87.5 \%$ closure in 11 eyes using autologous platelet concentrate. In this study, using $\mathrm{C}_{3} \mathrm{~F}_{8}$ gas tamponade and autologous platelet concentrate we had an anatomic success rate of $96 \%$. This was significantly better than our success rate using $\mathrm{SF}_{6}$ gas alone $(p=0.005)$, or $\mathrm{SF}_{6}$ gas and TGF- $\beta(p=0.017)$ Although our best results were obtained in the platelet concentrate group, we also used a longer acting gas tamponade in these eyes so a combination of these two factors may be responsible for the improved results. It is clear, however, that using a variety of techniques and adjuncts, it is now possible to obtain a very high anatomic success rate in the surgical treatment of macular holes. The precise role of adjunctive therapy and the various surgical techniques employed such as foveal manipulation can probably be addressed only within a controlled clinical trial.

In 11 eyes, despite resolution of the subretinal fluid, the edge or part of the edge of the macular hole was visible postoperatively. It is of interest that only two $(5 \%)$ of these were in the platelet treated group, compared to five (33\%) in the $\mathrm{SF}_{6}$ only group, and four $(40 \%)$ in the $\mathrm{SF}_{6}$ and $\mathrm{TGF}-\beta 2$ group. This difference between the platelet treated group and the others was statistically significant $(p=0.01)$. We saw this complication only rarely in eyes treated with platelets and $\mathrm{C}_{3} \mathrm{~F}_{8}$. This may indicate a more enhanced chorioretinal adhesive response in this group, compared with the other two groups. Whether this is due to the action of platelets, or due to the longer lasting tamponade, we cannot say; however, we intend to conduct a study to examine the effects of platelet concentrate using a shorter duration of tamponade. Although the cuff of subretinal fluid had resolved in these eyes, the presence of an edge may reduce the functional result. Eyes without an edge were more likely to achieve at least four lines of improvement than those with an edge $(p=0.01)$.

Closure of the hole is usually associated with an improvement in visual acuity. Patients benefit from surgery by an increase in visual acuity, and a resolution of metamorphopsia. Visual acuity of $6 / 12$ or better will enable patients to drive. Improved visual acuity with resolution of metamorphopsia will affect reading ability. Polk et $a l^{20}$ showed that there is an improvement in bilateral visual function following macular hole surgery, particularly if there is subnormal vision in the fellow eye. Most authors use a two line improvement in Snellen visual acuity as an indication of success; however, actual final visual acuity is, perhaps, more important.

Overall visual results in this study were similar to those in other reports, ${ }^{561218}$ with two line improvement achieved in $74 \%$ of eyes treated with $\mathrm{C}_{3} \mathrm{~F}_{8}+$ platelets. Glaser and colleagues ${ }^{6}$ also suggested that TGF- $\beta 2$, as well as having the properties of a 'biologic chorioretinal glue', ${ }^{16}$ may also have additional effects on visual function at a cellular level, on photoreceptor function and/or regeneration. There is a possibility that the release of cytokines from platelets may have an effect on local cellular function. We, however, found, no significant difference in visual acuity improvement between those groups receiving platelets and those receiving no adjunctive therapy.

We inject $0.1 \mathrm{ml}$ of a platelet solution containing $10^{6}$ platelets per $\mathrm{ml}$ into the eye. For the following 1-2 weeks, a white 'coagulum' is visible over the posterior fundus and almost always covering the macular hole. This may be merely clumping of the platelets, or the material may contain some clotting factorsfor example, fibrin. At present we are analysing 
the exact constituents of this material. Others working with platelets have also noted this appearance (Gaudric, personal communication). This white material may have a beneficial effect by enhancing closure of the macular hole by mechanical means, perhaps providing a scaffold for cell proliferation, but it is also possible that it has a deleterious effect on retinal tissue. A number of eyes treated with platelet concentrate, despite having successfully closed holes, had one of a variety of macular abnormalities. Twenty had retinal pigment abnormalities at the fovea. At about 6 weeks postoperatively, a hyperpigmentation of the retinal pigment epithelium occurred at the site of the macular hole in these 20 eyes. Surrounding this RPE spot the hole was closed with no edge visible. Seventeen of this group were treated with platelet concentrate and $\mathrm{C}_{3} \mathrm{~F}_{8}$ gas, two had $\mathrm{SF}_{6}$ gas tamponade, and one had $\mathrm{SF}_{6}$ gas and TGF- $\beta 2$. The platelet treated group was more likely than either of the other two groups to develop a pigment spot $(\mathrm{p}=0.048$ and $p=0.025$ respectively). Even with this pigment epithelium disturbance, the mean visual outcome, in these patients was not significantly different from those without the pigment spot $(p=0.27)$, and indeed one patient with postoperative visual acuity of $6 / 6$ had a pigment spot.

We noted radial striae emanating from the closed macular hole in 12 eyes postoperatively. They present postoperatively and then gradually disappear over about 6 weeks. Radial striae in the retina are present in some macular holes preoperatively and their presence has been correlated with a better visual outcome. ${ }^{21}$ We were unable to define any significant difference in visual outcome in our patients with radial folds but the numbers are quite few. The retina remains thickened at the fovea for several weeks postoperatively in some eyes in which the macular hole has closed and these striae may represent the effects of centripetal contraction on hole closure.

Our experience with TGF- $\beta 2$ was disappointing with a significantly worse visual outcome in this group than in either of the other two groups. Our numbers, however, are limited and a high proportion $(40 \%)$ of these eyes were reoperations with a substantial delay between first and second operation making visual improvement less likely. TGF- $\beta 2$ is extracted from porcine or bovine platelets. It is possible that microheterogeneities in the structure of the compound may affect its action and could explain our disappointing results. Although initially a $100 \%$ success rate was reported using $330 \mathrm{ng}$ or1330 $\mathrm{ng}$ of bovine TGF- $\beta 2$, in a more recently conducted controlled clinical trial comparing recombinant TGF- $\beta 2$ and placebo, no significant difference was found between the TGF- $\beta 2$ treated group and control group. ${ }^{22}$

Twenty eyes had stage 2 macular holes; 15 $(75 \%)$ of these had a final visual acuity of $6 / 18$ or better, and $12(60 \%)$ had a final acuity of $6 / 12$ or better. In this study, the mean postoperative visual acuity for stage 2 macular holes is $\log \operatorname{MAR} 0.5(6 / 18)$; it is $0.6(6 / 24)$ for stage 3 ; and $0.8(6 / 36)$ for stage 4 . Stage 2 holes, when successful, are more likely than either stage 3 $(\mathrm{p}=0.012)$ or stage $4(\mathrm{p}=0.004)$ to achieve a final visual acuity of $6 / 9$ or better. A previous study has also indicated a favourable outcome of stage 2 holes, and that patients with recent onset holes have a better visual prognosis than older holes. ${ }^{23}$ We have attempted to make sure that macular holes were present for a no longer than 1 year, but depending on patient symptoms, an exact measure of duration is sometimes difficult to ascertain.

It has been suggested that reoperation should be considered in cases in which the macular hole has failed to close at initial surgery. ${ }^{25}{ }^{26}$ In our series of eight reoperations, although six $(75 \%)$ were an anatomic success, only $25 \%$ achieved visual improvement of two lines or more. This may be due to a delay in the reoperation time, in our patients, ranging from 1.5 to 11 months (mean 7 months).

The recognised complications of macular hole surgery include cataract, retinal tears, retinal detachment, visual field loss, reopening of the hole, and optic atrophy. ${ }^{27}{ }^{28}$ We have experienced all of these complications. We had five $(6 \%)$ retinal tears, which were treated with cryotherapy intraoperatively. Three $(3.5 \%)$ eyes developed retinal detachments, two successfully repaired. One failure developed proliferative vitreoretinopathy. In two (2.5\%) patients a previously closed macular hole reopened, requiring repeat vitrectomy, one at about 4 weeks postoperatively and the other at 3 months. We also experienced one case of postoperative optic atrophy $(1.4 \%)$. The reason for this is unclear, there was no postoperative intraocular pressure rise and this patient was a healthy young man with no apparent systemic disease. This patient resembles the three patients described by Stirpe with optic atrophy following macular hole surgery ${ }^{29}$ and which he attributed to damage to the optic nerve fibre layer during stripping of the posterior hyaloid.

Endophthalmitis is a concern when injecting any material into the vitreous cavity, and has occurred following injection of serum as an adjunct in macular hole surgery ${ }^{30}$ (P E Liggett, personal communication). We believe that preparation of the platelet concentrate within the operating theatre avoiding transport of the blood or blood product may reduce the risk of bacterial or viral contamination.

Visual field loss has been described in association with vitrectomy ${ }^{31}$ and more recently following macular hole surgery. ${ }^{32}{ }^{33} \mathrm{We}$ found visual field defects in 22 eyes, 16 in the $\mathrm{C}_{3} \mathrm{~F}_{8}$ gas and platelet concentrate group, all of whom had preoperative and postoperative perimetry. The visual field loss was quite dense, peripheral, usually inferotemporal, and in many patients, symptomatic despite improvement in central vision. In a few eyes, the cause of the visual field defect was apparent-one had optic atrophy, one had anterior ischaemic optic neuropathy, and one had a retinal detachment. In the majority, the cause of the visual field loss is uncertain, but it appears to be due to retinal nerve fibre loss rather than outer retinal damage. ${ }^{32}$ It has been attributed 
to a variety of causes but especially to trauma to the optic nerve fibres during posterior hyaloid stripping, when optic nerve haemorrhages may occur. ${ }^{33}$ In this study, however, five patients with visual field loss did not have posterior hyaloid removal during surgery, three had stage 4 macular holes, and the other patient developed a visual field defect following a second procedure for previously failed surgery. It has also been suggested that surgical damage to the optic nerve head may occur during air/fluid exchange, ${ }^{31}$ although this is, perhaps, unusual with silicone tipped needles. Other factors that could possibly account for visual field loss include nocturnal hypotension causing relative ischaemia of the optic nerve, raised intraocular pressure, ocular compression during prone posturing, and the effects of the intraocular gas bubble. ${ }^{33}$ The flotation force exerted by the intraocular gas bubble on the retina is very low and probably of little clinical significance; nevertheless, it may exacerbate retinal ischaemia in susceptible patients and may, perhaps, account for the predominant pattern of inferior rather than superior visual field loss. In this study, eyes treated with $\mathrm{C}_{3} \mathrm{~F}_{8}$ gas tamponade were more likely to develop field defects than eyes treated with shorter acting $\mathrm{SF}_{6}$ gas although this difference failed to reach statistical significance. Raised intraocular pressure is not unusual immediately after macular hole surgery and it may exacerbate retinal ischaemia, and be implicated in the aetiology of visual field loss. To prevent it we routinely treated all patients with oral acetazolamide postoperatively, and the highest recorded postoperative intraocular pressure was $34 \mathrm{~mm} \mathrm{Hg}$. Of the 22 cases of field loss encountered in this study, at least six were symptomatic, so that we now inform patients of this risk before surgery.

In this study, we achieved a high rate of anatomic and visual success in the group of eyes treated with vitrectomy, gas tamponade, and autologous platelet concentrate. The methods used in this group differed from the other two groups not only in terms of adjunctive therapy but also by the avoidance of foveal manipulation and drainage of fluid through the hole, and by the use of a longer gas tamponade with a longer posturing requirement. One, or all, of these may have contributed to the improved success rate. Our best results were obtained in stage 2 macular holes. Our findings confirm previous pilot studies suggesting a beneficial outcome using a platelet concentrate as an adjunct in the surgical therapy of macular holes. ${ }^{8}$ Although many patients had a significant improvement in visual acuity, the incidence of postoperative visual field loss may be as high as $30 \%$. It is now possible to achieve an anatomic success rate of greater than $90 \%$ in the surgical treatment of macular holes using a variety of techniques including adjuvants such as platelet concentrate, serum, or cytokines or by carefully dissecting fine epiretinal membranes or the internal limiting membrane around the hole. All these techniques employ $\mathrm{C}_{3} \mathrm{~F}_{8}$ gas tamponade and prolonged postoperative posturing which is difficult and unsatisfac- tory for elderly patients. Efforts must now be directed to identify techniques which reduce the posturing requirement while maintaining the high anatomic and visual success rate in macular hole surgery.

The authors thank Dr K P Cotter and Mr N Martin,
Haematology Department, Cork University Hospital for their Haematology Department, Cork University Hospital for their
help in preparation and analysis of the autologous platelet concentrate.

1 The Eye Disease Case-Control Study Group. Risk factors for idiopathic macular holes. Am f Ophthalmol 1994; 118:754-61

2 Gass JDM. Idiopathic macular hole; its early stages and pathogenesis. Arch Ophthalmol 1988;106:629-39.

3 Lewis H, Cowan GM, Straatsma BR. Apparent disappearance of a macular hole with development of an epiretinal membrane. Am f Ophthalmol 1986;102:172-5.

4 Schocket SS, Lakhanpal V, Miao XP Laser treatment of macular holes. Ophthalmology 1988;95:574-82.

5 Kelly NE, Wendel RT. Vitreous surgery for idiopathic macular holes: results of a pilot study. Arch Ophthalmol 1991;109:654-9.

6 Glaser BM, Michels RG, Kuppermann BD, Sjaarda RN, Pena RA. Transforming growth factor-beta 2 for the treatment of full-thickness macular holes; a prospective ment of full-thickness macular holes; a prospect

7 Liggett PE, Skolik DSA, Horio B, Saito Y, Alfaro V, Mieler

Liggett PE, Skolik DSA, Horio B, Saito Y, Alfaro V, Mieler
W. Human autologous serum for the treatment of W. Human autologous serum for the treatment of
full-thickness macular holes. A preliminary study. Ophthalmology 1995;102:1071-6.

8 Gaudric A, Massin P, Paques M, Santiago PY, Guez JE, Le Gargasson JF, et al. Autologous platelet concentrate for the treatment of full-thickness macular holes. Graefes Arch Clin Exp Ophthalmol 1995;233:549-54.

9 Barrie T. Macular holes. (Editorial) $\mathrm{Br} f \mathrm{f}$ Ophthalmol 1995; 79:511-2

10 Fine SL. Vitreous surgery for macular hole in perspective: is there an indication? Arch Ophthalmol 1991;109:635-6.

11 Gass JDM. Reappraisal of biomicroscopic classification of stages of development of a macular hole. Am f Ophthalmol
1995;119:752-9.

12 Lansing MB, Glaser BM, Liss H, Hanham A, Thompson JT, Sjaarda RN, et al. The effect of pars plana vitrectomy JT, Sjaarda RN, et al. The effect of pars plana vitrectomy without epiretinal membrane peeling on full-

13 Thompson JT, Glaser BM, Sjaarda RN, Murphy RP, Hanham A. Effects of intraocular bubble duration in the treatment of macular holes by vitrectomy and transforming growth factor beta 2. Ophthalmology 1994;101:1195-200.

4 Steed DL, Gosler JB, Holloway GA, Malone JM, Bunt TJ, Webster MW. Randomised double-blind trial in treating chronic diabetic foot ulcers CT102 activated platelet supernatant, topical versus placebo. Diabetes Care 1992;15: 1598-604.

15 Korobelnik JF, Hannouche D, Belayachi N, Branger M, Guez JE, Hoang-Xuan T. Autologous platelet concentrate as an adjunct in macular hole healing. A pilot study. Ophthalmology 1996;103:590-4.

16 Smiddy WE, Glaser BM, Green WR, Concor TB, Roberts $\mathrm{AB}$, Lucas $\mathrm{R}$, et al. Transforming growth factor beta. A bioAB, Lucas R, et al. Transforming growth factor beta. A bio-
logic chorioretinal glue. Arch Ophthalmol 1989;107:57780 .

17 Christmas NJ, Skolik SA, Howard MA, Saito Y, Barnstable CJ, Liggett PE. Treatment of retinal breaks with autologous serum in an experimental model. Ophthalmology 1995;102: 263-71

18 Wendel RT, Patel AC, Kelly NE, Salzano TC, Wells JW, Novack GD. Vitreous surgery for macular holes. Ophthalmology 1993;100:1671-6.

19 Smiddy WE, Glaser BM, Thompson JT, Sjaarda RN, Flynn HW, Hanham A, et al. Transforming growth factor beta 2 significantly enhances the ability to flatten the rim of subretinal fluid surrounding macular holes. Preliminary results of a multicenter prospective randomised study. Retina 1993;13:296-301.

20 Polk TD, Smiddy WE, Flynn HW. Bilateral visual function after macular hole surgery. Ophthalmology 1996;103:422-6. 21 Yoshida A, Ishiko S, Akiba J. Radiating retinal folds detected Yoshida A, Ishiko S, Akiba J. Radiating retinal folds detected
by the scanning laser ophthalmoscope in idiopathic macuby the scanning laser ophthalmoscope in idiopathic macu-
lar holes. Report at Club Jules Gonnin Meeting; September lar holes.

22 Thompson JT, Smiddy WE, Williams GA. Comparison of recombinant TGF-B2 and placebo as an adjunctive agent for macular hole surgery. Ophthalmology 1995; 102(suppl): 91.

23 Ruby AJ, Williams DF,Grand MG, Thomas MA, Meredith TA, Boniuk I, et al. Pars plana vitrectomy for treatment of stage 2 macular holes. Arch Ophthalmol 1994;112:359-64.

24 Ryan EH, Gilbert HD. Results of surgical treatment of recent-onset full thickness macular holes. Arch Ophthalmol 1994;112:1545-53.

25 Ie D, Glaser BM, Thompson JT, Sjaarda RN, Gordon LW. Retreatment of full-thickness macular holes persisting after prior vitrectomy. A pilot study. Ophthalmology 1993;100: prior vitrect

26 Smiddy WE, Sjaarda RN, Glaser BM, Flynn HW, Thompson JT, Hanham A, et al. Reoperation after failed macular hole surgery. Retina 1996;16:13-18. 
27 Thompson JT, Glaser BM, Sjaarda RN, Murphy RP. Progression of nuclear sclerosis and long-term visual results of vitrectomy with transforming growth factor beta-2 for macular holes. Am $f$ Ophthalmol 1995

28 Park SS, Marcus DM, Duker JS, Pesavento RD, Topping TM, Frederick AR, et al. Posterior segment complication after vitrectomy for macular hole. Ophthalmology 1995;102: $775-81$.

29 Stirpe M. Complications of removal of the posterior vitreous face during vitreous surgery. Presented at Club Jules Gonin Meeting, September 1996.
30 Bacon AS, Davison CR, Patel BC, Frazer DG, Ficker LA, Dart JKG. Infective endophthalmitis following vitreoretinal surgery. Eye 1993;7:529-34.

31 Melberg NS, Thomas MA. Visual field loss after pars plana vitrectomy with air/fluid exchange. Am F Ophthalmol 1995; 120:386-8.

32 Ezra E, Arden GB, Riordan-Eva P, Aylward GW, Gregor ZJ.Visual field loss following vitrectomy for stage 2 and 3 macular holes. Br f Ophthalmol 1996;80:519-25.

33 Prendergast SD, McCuen BW. Visual field loss after macular hole surgery Ophthalmology 1996;103:1069-77. 